\title{
PRP treatment effects on degenerative tendinopathy - an in vitro model study
}

\author{
Jianying Zhang \\ James H-C. Wang
}

MechanoBiology Laboratory, Departments of Orthopaedic Surgery, Bioengineering, and Mechanical Engineering and Materials Science, University of Pittsburgh, PA

Corresponding author:

James H-C. Wang

210 Lothrop Street, BST, E1640

15213 Pittsburgh, PA

E-mail:wanghc@pitt.edu

\section{Summary}

Platelet-rich plasma (PRP) has become a popular option for the treatment of injured tendons. However, the efficacy of PRP treatment is a matter of heated debate in orthopaedics and sports medicine. In this study, we used a cell culture model to evaluate the potential effects of PRP treatment on degenerative tendinopathy. The in vitro model, which uses the current concept of "diseases-in-adish", consisted of tendon stem/progenitor cells (TSCs) that were derived from rabbit tendons and cultured in differentiating media with and without autologous platelet-rich clot releasate (PRCR). We found that $10 \%$ PRCR treatment of TSCs blocked their non-tenogenic differentiation, as evidenced by the marked decrease in lipid droplets, proteoglycan accumulation, and calcium deposition on cell surfaces. Moreover, the protein markers for non-tenocytes (adiponectin, collagen type II, and osteocalcin) were either minimally expressed or greatly reduced. However, after TSCs underwent non-tenogenic differentiation by pre-treatment in non-tenogenic media for two days, PRCR only slightly reduced adipogenesis and osteogenesis of TSCs, although chondrogenesis was markedly suppressed. Finally, PRCR treatment after pretreatment of TSCs in non-tenogenic media for one week had little effect on any of the three nontenogenic differentiations of TSCs. These findings suggest that the injection of PRP in clinics may not be able to effectively reverse the degenerative conditions of late-stage tendinopathy, which are characterized by lipid depositions, proteoglycan accumulation, and calcification, either alone or in combination.
KEY WORDS: degenerative tendinopathy, PRP, tendon stem cells, disease-in-a-dish-model.

\section{Introduction}

Tendinopathy is a highly prevalent tendon disorder in both occupational and athletic settings ${ }^{1,2}$. In advanced stages, tendinopathy is manifested by the formation of lipids, proteoglycans, and calcified tissues in tendon lesions ${ }^{3}$. While a number of etiological factors, including aging, genetics, and mechanical loading 4,5 , have been implicated in the development of tendinopathy, the precise pathogenesis of tendinopathy remains elusive. As a result, current treatments of tendinopathy are largely empirical, and recurrence of the tendon disorder is common ${ }^{5}$.

Towards better understanding the cellular mechanism of tendinopathy, intensive research in recent years has focused on characterizing tendon cells. It is now known that, in addition to consisting of tenocytes, the dominant tendon cells responsible for tendon maintenance, repair, and remodeling, tendons also contain a new type of tendon cells, termed tendon stem/progenitor cells $(\mathrm{TSCs})^{6-9}$. Unlike tenocytes, TSCs possess multi-differentiation potential ${ }^{7}$; at early stages, these tendon-specific stem cells express stem cell markers Oct-4 and SSEA-4, among others ${ }^{7}$. In addition, we further established that under excessive mechanical loading conditions, TSCs can differentiate into non-tenocytes (adipocytes, chondrocytes, and osteocytes) in addition to tenocytes ${ }^{10}$. These diverse phenotypes of differentiated TSCs match well with the non-tendinous tissues (lipids, accumulated proteoglycans, and calcified tissues) seen in the lesions of tendinopathic tendons ${ }^{3}$. Therefore, TSCs could play a major role in the development of tendinopathy by differentiating aberrantly into non-tenocytes ${ }^{10}$.

As adult stem cells, TSCs in vivo are localized in "niches"6, which are specialized tendon microenvironments. These niches are critical for tendon homeostasis and pathophysiology, as they regulate TSCs' ability to maintain healthy tendons and repair injured tendons, such as in the case of tendinopathy. One vital niche factor for TSCs is a family of growth factors in tendons, including PDGF, EGF, TGF- $\beta 1$, IGF-I, VEGF, and bFGF, which are involved in tendon healing ${ }^{11-14}$. This pool of growth factors that stimulate tendon healing provides a rationale for using autologous platelet-rich plasma (PRP), a natural source of growth factors, to treat injured tendons. PRP treatment is now widely used in clinics to enhance healing of injured tissues including tendons and ligaments ${ }^{15,16}$. 
However, clinical trials have not yet established a clear consensus on the efficacy of PRP treatment for tendinopathy ${ }^{17-19}$. Such variability in the effects of PRP treatment highlight the difficulty of interpreting data from human patient studies, where many factors, including age, gender, disease and treatment history, and subjective evaluation of treatment effects, come into play. Thus, basic scientific studies that afford more rigorous study design and enable more objective evaluation of the PRP treatment effects are highly desirable.

In this study, our goal was to assess the effects of PRP treatment on degenerative tendinopathy in vitro. To this end, we cultured TSCs under various differentiating conditions as an in vitro "tendinopathy" model, based on the premise that TSCs are the major cell type that contributes to the pathogenesis of degenerative tendinopathy 20 and that PRP treatment improves tendon's degenerative conditions by "correcting" nontenogenic differentiation of TSCs. The use of TSCs as an in vitro tendinopathy model reflects the current concept of using stem cells as a "diseases-in-a-dish" mod$\mathrm{el}^{21,22}$ and allows objective evaluation of the results.

\section{Materials and methods}

\section{Preparation of PRP-clot releasate (PRCR)}

The protocols for collecting blood samples and tendon tissues (below) from rabbits were approved by the University of Pittsburgh IACUC ${ }^{23}$.

Whole blood obtained from nine young adult New Zealand white rabbits (8-10 months old, 3.0-4.0 kg) was used to prepare PRP-clot releasate (PRCR) according to our previously published protocol ${ }^{24}$. Briefly, 7-9 $\mathrm{ml}$ whole blood was centrifuged at $400 \mathrm{~g}$ for 15 min, and the supernatant containing highly concentrated platelets was collected and termed as PRP. Then, platelets in PRP were activated by incubating with $22 \mathrm{mM} \mathrm{CaCl} \mathrm{mat}_{2}$ at $37^{\circ} \mathrm{C}$ for $1 \mathrm{hr}$, followed by centrifugation at $2,000 \mathrm{~g}$ for $10 \mathrm{~min}$. The final supernatant, referred to as PRCR, was collected by aspiration and stored at $4^{\circ} \mathrm{C}$ until further use. This method yielded PRP containing no buffy coat products and about 3.2-fold concentration of platelets over the baseline value of whole rabbit blood. Also, the PRCR preparation method was similar to that reported in a previous study 25 .

\section{Cell culture experiment}

TSCs from the patellar tendons of the rabbits mentioned above were obtained based on our previously described methods ${ }^{7,24}$. They were cultured in growth medium (DMEM + 20\% FBS) for $24 \mathrm{hrs}$ and TSCs at passage 1 were plated on a 6 -well plate at a density of $2.4 \times 10^{5}$ cells/well in regular growth medium (DMEM + $10 \%$ FBS). After 2 days, the regular growth medium was replaced with adipogenic, chondrogenic, or osteogenic differentiation media with or without $10 \%$
PRCR. The choice of PRCR concentration was based on the marked effects caused by $10 \%$ PRCR on TSC proliferation and differentiation ${ }^{24}$. In a parallel experiment, two groups were maintained: i) TSCs were cultured in non-tenogenic media for 2 days, then PRCR was added and TSCs cultured for 3 additional days; ii) TSCs were grown in non-tenogenic media for 7 days, then treated with PRCR for 7 more days. The effects of PRCR treatment on TSC differentiation were then assessed in both groups by gene expression analysis.

The non-tenogenic differentiation media used in culture experiments were described previously ${ }^{7}$. The adipogenic induction medium consisted of regular growth medium with dexamethasone $(1 \mu \mathrm{M})$, insulin $(10 \mu \mathrm{g} / \mathrm{ml})$, indomethacin $(100 \mu \mathrm{M})$, and isobutylmethylxanthine $(0.5 \mathrm{mM})$; the chondrogenic induction medium was made by mixing regular growth medium with proline $(40 \mu \mathrm{g} / \mathrm{ml})$, dexamethasone $(39 \mathrm{ng} / \mathrm{ml})$, TGF- $\beta 3$ (10 ng/ml), ascorbate 2-phosphate (50 $\mu \mathrm{g} / \mathrm{ml})$, sodium pyruvate $(100 \mu \mathrm{g} / \mathrm{ml})$, and insulintransferrin-selenious acid mix $(50 \mathrm{mg} / \mathrm{ml})$. Finally, osteogenic induction medium was made by supplementing regular growth medium with dexamethasone $(0.1$ $\mu \mathrm{M})$, ascorbic 2-phosphate (0.2 mM), and glycerol 2phosphate $(10 \mathrm{mM})$.

\section{Assessing non-tenocyte differentiation of TSCs}

Oil Red O assay, Safranin O assay, and Alizarin Red S assay were used to assess adipogenesis, chondrogenesis, and osteogenesis of TSCs, respectively ${ }^{7}$. Briefly, TSCs cultured in adipogenic medium were fixed in $4 \%$ paraformaldehyde and incubated in $0.36 \%$ Oil Red $\mathrm{O}$ solution (Millipore, Cat. \# 90358) to visualize positive cells containing lipid droplets. Cells cultured in chondrogenic medium were fixed in ice-cold $75 \%$ ethanol followed by staining with Safranin O solution (Sigma, St. Louis; Cat. \# HT904). Finally, cells grown in osteogenic medium were fixed in chilled $70 \%$ ethanol and stained with Alizarin Red S (Millipore, Cat. \# 2003999). Stained cells were examined on an inverted microscope (Nikon eclipse, TE2000-U), and images were taken by a CCD camera on the microscope and analyzed by SPOT ${ }^{\mathrm{TM}}$ imaging software.

\section{Immunostaining of TSCs and differentiated cells}

Immunostaining for the stem cell markers Oct-4 and SSEA-4, and the non-tenocyte markers adiponectin (adipocytes), collagen type II (chondrocytes), and osteocalcin (osteocytes), was performed using methods described previously ${ }^{7}$. In brief, Oct-4 staining was performed by first fixing TSCs from the different groups in $4 \%$ paraformaldehyde, and then sequentially incubating with rabbit anti-mouse Oct-4 (1:350) and Cy3-conjugated donkey anti-rabbit IgG antibodies (1:500). Staining for SSEA-4 was performed by incubating fixed TSCs with FITC-conjugated mouse antiSSEA-4 (1:250). Stained cells were examined under a fluorescence microscope and images of cells were 
obtained. All antibodies were from Abcam, Rockland, Millipore, or Santa Cruz Biotechnology.

\section{Western blot analysis}

Expression levels of adiponectin, collagen type II, and osteocalcin were determined on western blots performed according to previously described protocols $^{24}$. In brief, proteins were extracted from cells after each treatment using the M-PER mammalian protein extraction reagent (Thermo Scientific, Rockford, IL) followed by separation on $12 \%$ sodium dodecyl sulfate-polyacrylamide gel electrophoresis (SDSPAGE) gel. After transfer to Nitrocellulose membranes (Invitrogen, Carlsbad, CA) using a semi-dry blotting apparatus (Bio-Rad, Hercules, CA), adiponectin, Collagen type II and osteocalcin were detected using respective antibodies. To determine equal loading of proteins on the gels, GAPDH was probed using mouse anti-GAPDH antibody (1:500, Abcam, Cambridge, MA) and detected using the goat anti-mouse IgG-HRP antibody (1:1000, Santa Cruz, Dallas, TX). The positive bands were visualized on Xray films using the ECL Western Blotting Analysis System (GE Healthcare, Buckinghamshire, HP, UK).

\section{Quantitative RT-PCR (qRT-PCR)}

The procedures for qRT-PCR analysis have been described previously ${ }^{7}$. Briefly, RNA was extracted from cells in each treatment group using the RNeasy Mini Kit (Qiagen, Valencia, CA). Then, first-strand cDNA was reverse transcribed from $1 \mu \mathrm{g}$ total RNA using SuperScript II (Invitrogen, Grand Island, NY) with the following conditions: $65^{\circ} \mathrm{C}$ for $5 \mathrm{~min}$, followed by cooling at $4^{\circ} \mathrm{C}$ for $1 \mathrm{~min}$, reverse transcription at $42^{\circ} \mathrm{C}$ for $50 \mathrm{~min}$, and finally $72^{\circ} \mathrm{C}$ for $15 \mathrm{~min}$. qRT-PCR was performed using QuantiTect SYBR Green PCR Kit (QIAGEN, Valencia, CA) in a Chromo 4 Detector (MJ Research, St. Bruno, Quebec, Canada) using $2 \mu \mathrm{l}$ cDNA (total 100 ng RNA) and the following incubation times: $94^{\circ} \mathrm{C}$ for $5 \mathrm{~min}, 30$ to 40 cycles of $1 \mathrm{~min}$ at $94^{\circ} \mathrm{C}, 40$ seconds at $57^{\circ} \mathrm{C}$, and 50 seconds at $72^{\circ} \mathrm{C}$. A final extension at $70^{\circ} \mathrm{C}$ was performed for another $10 \mathrm{~min}$. Each reaction was replicated at least three times. The sequences of forward and reverse primers, and the amplified PCR products are detailed previously $7,26,27$. All primers were synthesized by Invitrogen. Relative gene expression levels were expressed as $2^{-\Delta \Delta C T}$, where CT is the cycle threshold value of each RNA sample amplified, and $\Delta \Delta C T$ was determined by the formula: $\Delta \Delta C T=\left(C_{\text {target }} / C T-\right.$ GAPDH $)_{\text {experiment }}-\left(\mathrm{CT}_{\text {target }} / \mathrm{CT}_{\mathrm{GAPDH}}\right)_{\text {control. }}$

\section{Statistical analysis}

An un-paired $t$-test was used to compare two groups (e.g. an experimental group vs its control group). The difference between the two groups was considered statistically significant when P-value was less than 0.05 .

\section{Results}

\section{PRCR promotes TSC differentiation into teno- cytes}

When cultured in growth medium (DMEM+20\% FBS), tendon cells extracted from rabbit patellar tendons exhibited a cobblestone-like morphology at confluence (Fig. 1A). They also expressed stem cell markers Oct-4 and SSEA-4 (Fig. 1B), confirming that these cells used in subsequent experiments were indeed TSCs at early stages. However, when TSCs were grown in regular growth medium (DMEM + 10\% FBS) supplemented with $10 \%$ PRCR for 7 days, the cells became highly elongated; this is a typical fibroblast-like phenotype (Fig. 1C), indicating induction of cell differentiation by PRCR. Moreover, the differentiated TSCs expressed high levels of tenocyte-related genes collagen types I and III, tenascin C, and tenomodulin, but not non-tenocyte related genes, including PPAR $\gamma$ (adipocyte), SOX-9 (chondrocyte), and Runx2 (osteocyte) (Fig. 1D). Collectively, these results indicate that PRCR treatment induced TSCs to differentiate into tenocytes.

\section{PRCR suppresses non-tenocyte differentiation of TSCs}

Since PRCR treatment of TSCs induced tenocyte differentiation in regular growth medium, we evaluated the effects of a similar treatment on TSC differentiation in three different non-tenogenic media. We found that when grown in adipogenic medium, TSCs differentiated into adipocytes, as evidenced by the formation of abundant lipid droplets on cell surfaces (Fig. 2A) and the expression of high levels of adiponectin (Fig. 2B), a protein specific for adipocytes. In contrast, when grown in the adipogenic medium with PRCR, both lipid production and adiponectin expression were markedly suppressed (Fig. 2C, D). Western blot analysis further confirmed the suppressive effect of PRCR treatment, as shown by the almost complete absence of adiponectin expression (Fig. 2E).

When TSCs were grown in chondrogenic medium, the cells differentiated into chondrocytes, as evidence by the high expression of proteoglycans (Fig. 3A) and collagen type II (Fig. 3B) on cell surfaces. However, when PRCR was present in the culture, expression of both proteoglycans and collagen type II markedly decreased (Fig. 3C, D). This PRCR-induced reduction in collagen type II expression levels was further verified by Western blot analysis (Fig. 3E).

Similar results were obtained when TSCs were cultured in the osteogenic induction medium with PRCR; with osteogenic medium alone, calcium deposits were abun- 

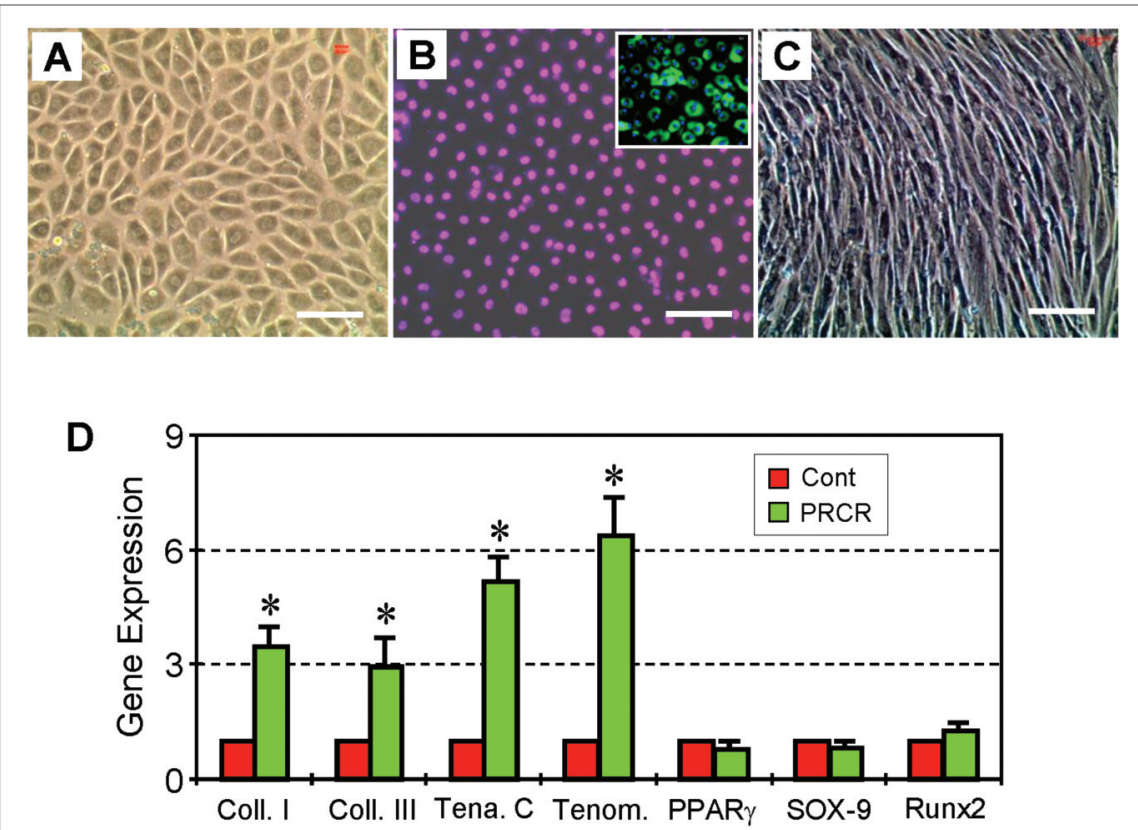

Figure 1. Verification of TSCs in culture and their ability to differentiate into tenocytes in regular growth medium. A. TSCs in culture exhibited a cobblestone shape. B. TSCs expressed stem cell markers Oct-4 (pink) and SSEA-4 (inset, green). C. TSCs differentiated into tenocytes in growth medium (DMEM + 10\% FBS) supplemented with $10 \%$ PRCR. The differentiated TSCs were highly elongated at confluence and assumed a typical fibroblast-like morphology. D. Gene expression of tenocyte and non-tenocyte associated genes in TSCs cultured in DMEM $+10 \%$ FBS (Cont) or in DMEM $+10 \%$ FBS $+10 \%$ PRCR (PRCR). TSCs that differentiated after PRCR treatment expressed high levels of tenocyte related genes (Coll. I, or collagen type I; Coll. III, or collagen type III; Tena. C, or tenascin C; and Tenom, or tenomodulin) but minimal levels of nontenocyte related genes PRAR $\gamma$, SOX-9, and Runx2 ( ${ }^{*} P<0.05$, respective to corresponding controls). Therefore, these highly-elongated, differentiated TSCs were classified as tenocytes. Bar: $100 \mu \mathrm{m}$.

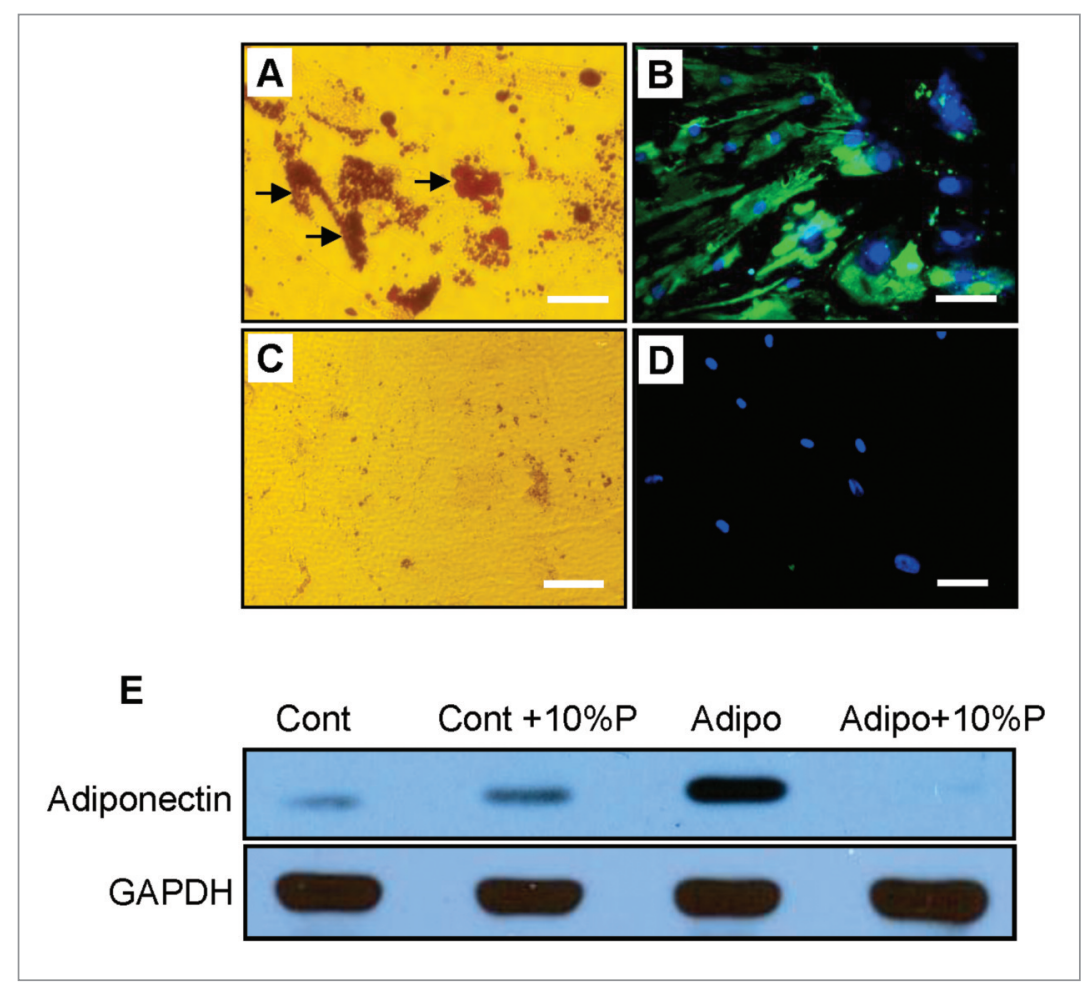

Figure 2. The effect of PRCR treatment on TSC adipogenesis. A, B. TSCs cultured in adipogenic medium alone. Abundant lipid droplets formed on the cell surface (A, arrows), and high levels of adiponectin were expressed (B, green). C, D. TSCs cultured in adipogenic medium with PRCR. The production of lipid droplets (C) and adiponectin expression (D) were markedly reduced by PRCR treatment. Blue represents nuclei stained with Hoechst 33342. E. Western blot analysis further demonstrated that PRCR treatment almost completely suppressed adiponectin expression (Cont - TSCs in DMEM + 10\% FBS; Cont+10\%P - TSCs grown in DMEM + 10\% FBS + 10\% PRCR; Adipo - TSCs cultured in adipogenic medium; Adipo+10\%P TSCs in adipogenic medium with 10\% PRCR). Three independent Western blot analyses were performed and similar results were obtained. Bar: $100 \mu \mathrm{m}$. 
dant on cell surfaces (Fig. 4A), and the osteocyte specific protein, osteocalcin, was also highly expressed (Fig. 4B). In contrast, when cultured with PRCR, calcium deposits were greatly reduced (Fig. 4C), and osteo- calcin expression also decreased markedly (Fig. 4D). Western blots also showed that PRCR treatment almost completely suppressed the osteocalcin expression induced by the osteogenic medium (Fig. 4E).
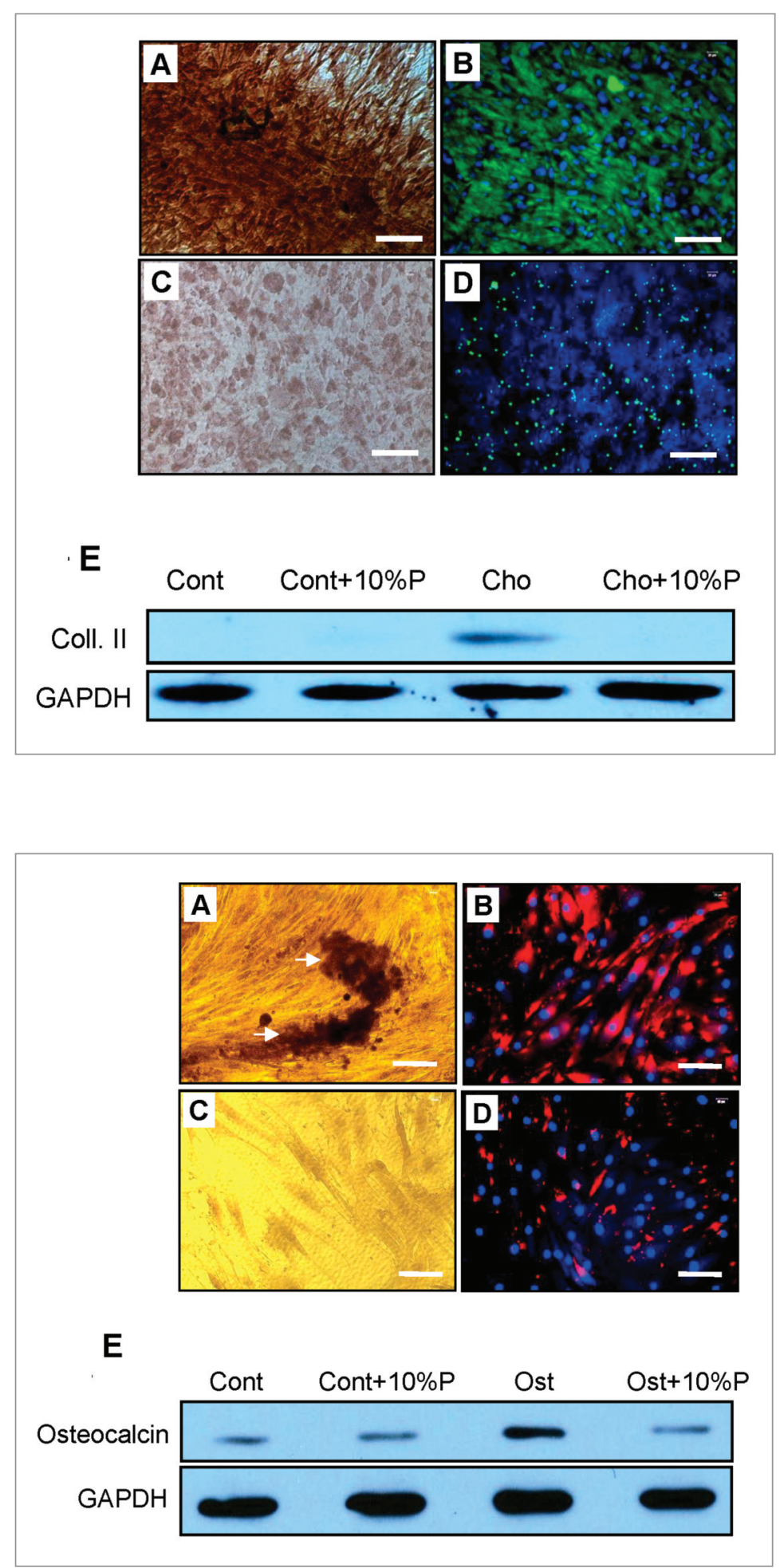

Figure 3. The effect of PRCR treatment on chondrogenesis of TSCs. A, B. When TSCs were grown in chondrogenic medium, they differentiated into chondrocytes, as shown by staining of abundant proteoglycans ( $\mathbf{A}$, brown) and expression of collagen type II (B, green). C, D. When 10\% PRCR was added to chondrogenic cultures, chondrogenesis was largely inhibited, as shown by minimal staining of proteoglycans (C) and collagen type II (D). Blue indicates nuclei stained with Hoechst 33342. E. Western blotting further confirmed that collagen type II expression was greatly reduced (ContTSCs in DMEM + 10\% FBS; Cont+10\%P TSCs grown in DMEM $+10 \%$ FBS $+10 \%$ PRCR; Cho - TSCs cultured in chondrogenic medium; Cho+10\%P - TSCs in chondrogenic medium with $10 \%$ PRCR). Similar results were obtained in three independent Western blot analyses performed. Bar: 100 $\mu \mathrm{m}$.
Figure 4. The effect of PRCR treatment on osteogenesis of TSCs. A, B. When grown in osteogenic medium, TSCs differentiated into osteocytes, which produced abundant calcium deposits on cell surfaces (A, arrows) and increased expression of osteocalcin (B, red). C, D. When 10\% PRCR was present in osteogenic cultures, the osteogenesis of TSCs was largely inhibited, as indicated by the minimal staining of calcium deposits (C) and osteocalcin expression (D). Nuclei were stained blue with Hoechst 33342. E. Osteocalcin expression was further shown to be largely inhibited in Western blot analyses. (Cont - TSCs in DMEM + $10 \%$ FBS; Cont $+\mathbf{1 0} \%$ P. TSCs grown in DMEM + 10\% FBS + 10\% PRCR; Ost TSCs cultured in osteogenic medium; Ost+10\%P - TSCs in osteogenic medium with $10 \%$ PRCR). Similar results were obtained from three independent Western blot experiments. Bar: $100 \mu \mathrm{m}$. 


\section{PRCR effects on non-tenogenic differentiation of TSCs depend on differentiation stage}

Finally, we determined whether PRCR treatment exerted similar suppressive effects on non-tenocyte differentiation of TSCs after pre-treatment in adipogenic, chondrogenic, or osteogenic medium followed by the addition of PRCR at specific time points. We found that when TSCs had undergone non-tenogenic differentiation for 2 days, PRCR treatment was able to slightly reduce adipogenesis and osteogenesis, as evidenced by subtle differences in the expression of PPAR $\gamma$ and Runx2 genes, respectively. In contrast, chondrogenesis, marked by SOX-9 expression, was suppressed significantly by PRCR (Fig. 5). However, after TSCs were cultured in non-tenogenic differentiation media for 7 days, the suppressive effects of PRCR treatment on all three non-tenocyte differentiations became small (Fig. 6).

\section{Discussion}

PRP treatment is widely used in orthopaedics and sports medicine to treat tendinopathy. However, basic scientific studies on its efficacy to justify the use of $\mathrm{PRP}$ in clinics are lagging behind ${ }^{28}$. Using an in vitro model, this study determined the effects of PRP treatment on the differentiation of TSCs in well-controlled tenogenic and non-tenogenic environments. We first verified the identity of TSCs by the expression of stem cell markers, Oct-4 and SSEA-4, and their ability to differentiate into tenocytes in regular culture medium with PRCR treatment. Demonstrating TSCs

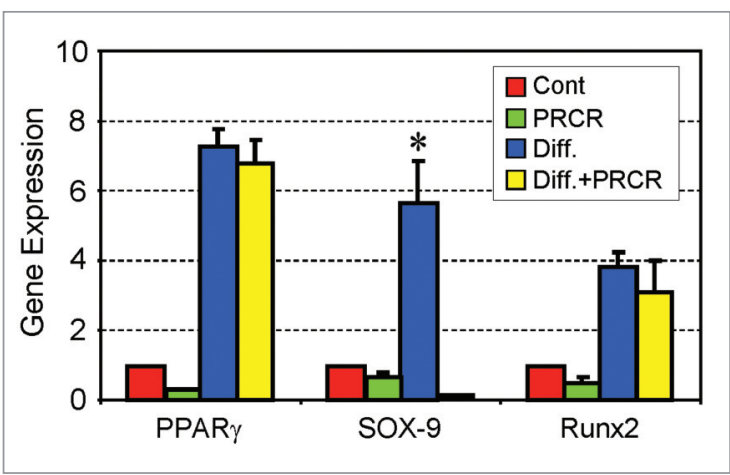

Figure 5. The effects of PRCR treatment on non-tenocyte differentiation of TSCs 2 days after growth in nontenogenic medium. PRCR treatment at $10 \%$ concentration for an additional 3 days only marginally decreased adipogenesis (PPAR $\gamma$ ) and osteogenesis (Runx2) of TSCs; however, chondrogenesis of TSCs (SOX-9) was drastically reduced by PRCR treatment ( $\mathrm{P}<0.05$, with respect to chondrogenic medium control). Note that the SOX-9 expression in the PRCR treated group is barely visible. (Cont - TSCs in DMEM + 10\% FBS; PRCR - TSCs grown in DMEM + 10\% FBS + 10\% PRCR; Diff. - TSCs cultured in adipogenic (PPARY), chondrogenic (SOX-9), or osteogenic (Runx2) media; Diff.+PRCR - TSCs in adipogenic, chondrogenic, or osteogenic media with $10 \%$ PRCR).

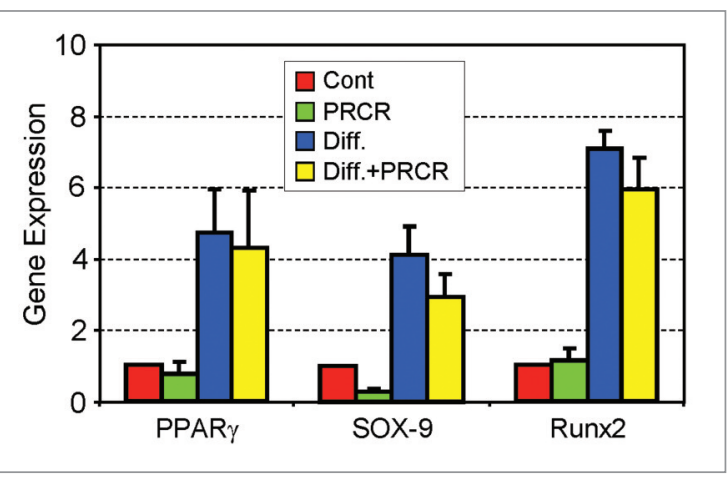

Figure 6. The effects of PRCR treatment on non-tenocyte differentiation of TSCs after pre-treatment with nontenogenic media for 7 days. PRCR treatment at $10 \%$ concentration for an additional 7 days only slightly decreased non-tenocyte differentiations (adipogenesis, chondrogenesis, and osteogenesis) of TSCs. (Cont - TSCs in DMEM + $10 \%$ FBS; PRCR - TSCs grown in DMEM + 10\% FBS + 10\% PRCR; Diff. - TSCs cultured in adipogenic (PPARY), chondrogenic (SOX-9), or osteogenic (Runx2) media; Diff.+PRCR - TSCs in adipogenic, chondrogenic, or osteogenic media with $10 \%$ PRCR).

identity in culture is critical for this study for the following reason: our in vitro TSC culture is a model for clinical degenerative tendinopathy, which is characterized by lipid depositions, proteoglycan accumulation, and calcification in affected tendons. These cultured TSCs indeed exhibited characteristics similar to those observed in degenerative tendinopathic tendons, which include high expression levels of both non-tenocyte related genes, such as PPAR $\gamma$, SOX-9, and Runx2, and non-tenocyte associated proteins, such as adiponectin, collagen type II, and oesteocalcin, which result in lipid depositions, proteoglycan accumulation, and calcification, respectively. It has been suggested that a TSC-based mechanism of degenerative tendinopathy involves the aberrant differentiation of TSCs into non-tenocyte lineages, which result in the formation of adipogenic, chondrogenic, and oesteogenic tissues ${ }^{29}$.

We next showed that the presence of PRCR in adipogenic, chondrogenic, and osteogenic media effectively suppressed TSCs differentiation into respective non-tenocytes by the reduced levels of adiponectin (adipocytes), collagen type II (chondrocytes), and osteonectin (osteocytes). Further, addition of PRCR even after non-tenocyte differentiation of TSCs had already begun did not exacerbate the unfavorable differentiation (Figs. 5, 6). These findings suggest that in clinical practice, PRCR released from activated PRP could deter the differentiation of TSCs into nontenocyte lineages in addition to promoting differentiation of TSCs into tenocytes. Thus, PRP treatment in vivo is not likely to negatively affect injured tendons, but could even improve the tendinopathic tendons by preventing further non-tenocyte differentiation that may lead to the formation of fatty tissue, proteoglycans, and calcified tissues. 
Interestingly, while PRCR treatment did not greatly suppress adipogenesis and osteogenesis, it drastically decreased chondrogenesis of TSCs when added during early stages (2 days) of TSC differentiation (Fig. 5). The molecular mechanisms governing this specific function are unknown, and must be investigated further. This finding suggests that a preliminary screening procedure can be adopted in clinics to determine the "type" of tendinopathy before application of PRP treatment. Specifically, if a patient's tendinopathy mainly involves early accumulation of proteoglycans only, then PRP treatment could be used to deter disease progression due to its ability to markedly inhibit chondrogenesis of TSCs. If the patient's tendinopathy involves lipid accumulation, calcification, or both, then PRP treatment may not be effective in improving the disorder because PRCR treatment only slightly reduced both adipogenesis and osteogenesis of TSCs (Fig. 5). However, for any type of fully-developed or advanced stage tendinopathy, which are characterized by lipid deposition, calcification, and proteoglycan accumulation, PRP treatment may not be effective in improving tendon conditions in vivo. This argument is supported by the finding that PRCR did not significantly suppress non-tenocyte differentiation of TSCs when added to the non-tenogenic media after 7 days of culture, after non-tenocyte differentiation was well under way (Fig. $6)$. This suggests that while PRP treatment by injection can relieve tendon inflammation-induced pain, as shown by our recent study ${ }^{30}$, it may not be an effective treatment method because it will not reverse the already-differentiated tissues within the degenerated tendons, including lipid, cartilage-like, and calcifiedtissues. One alternative method to address this issue is to perform tendon debridement that involves the surgical removal of degenerated non-tendinous tissues, which is done in clinical practices ${ }^{31,32}$. This procedure could improve the tenogenic environment and thereby encourage stem cells such as TSCs to differentiate into tenocytes instead of non-tenocytes.

This study also has a few limitations; first, we used TSCs from young adult rabbits. It is known that aging reduces the number of TSCs in tendons and promotes non-tenocyte differentiation of $\mathrm{TSCs}^{9}$. Therefore, aging not only predisposes tendons to tendinopathy, but also diminishes the effects of PRP treatment on tendinopathic tendons due to: 1) fewer TSCs being present in aged tendons and hence available for PRCR to act on; and 2) the inability of PRCR to reverse non-tenogenic differentiation of TSCs that is already well on its way. Second, only one concentration of PRCR was used in this study. Additional studies are needed to determine whether higher or lower concentrations of PRCR exert different effects on TSC differentiation in nontenogenic media. Third, while PRCR treatment was shown to suppress non-tenogenic differentiation of TSCs, the precise factors in PRCR responsible for these suppressive effects remain undetermined. Fourth, since this is a cell culture model, many in vivo factors were not taken into account. Importantly, the collagen matrix surrounding TSCs in vivo, and mechanical loading, which is an inherent part of the ten- don environment, were absent in this model. Mechanical loading, however, is known to affect TSC differentiation ${ }^{10}$, and eccentric loading on Achilles tendons is also reported to exert beneficial effects on tendinopathic tendons ${ }^{33}$. Therefore, a refined in vitro model that includes mechanical loading should be devised to study possible additive or synergistic effects of both PRCR and mechanical loading in future studies. Finally, animal studies that afford rigorous study designs may be required to define the precise effects of PRP treatment in vivo on TSCs as well as the TSC-based mechanisms of tendinopathy.

In summary, this study shows that PRCR treatment induced differentiation of TSCs into tenocytes under regular culture conditions. Moreover, while PRCR treatment blocked chondrogenesis and slightly reduced adipogenesis and osteogenesis of TSCs at early stages of cell differentiation, at later stages, PRCR treatment exerted only small effects on suppressing non-tenogenic differentiation of TSCs. Overall, the findings of this study suggest that while PRP treatment is not likely to worsen tendinopathic conditions in vivo, PRP treatment by injection, which is a common practice in clinics, does not likely reverse the degenerative conditions (lipid deposition, accumulated proteoglycans, and calcification) of tendinopathic tendons in advanced stages.

\section{Acknowledgements}

Funding for this work from NIH under award numbers AR049921, AR060920, and AR061395 (JHW) is gratefully acknowledged. We also thank Dr. Nirmala Xavier for her assistance in the preparation of this manuscript.

\section{References}

1. Khan KM, Maffuli N. Tendinopathy: an Achilles' heel for athletes and clinicians. Clin J Sport Med. 1998; 8:151-154.

2. Almekinders LC, Temple JD. Etiology, diagnosis, and treatment of tendonitis: an analysis of the literature. Med Sci Sports Exerc. 1998;30:1183-1190.

3. Kannus P, Jozsa L. Histopathological changes preceding spontaneous rupture of a tendon. A controlled study of $891 \mathrm{pa-}$ tients. J Bone Joint Surg Am. 1991;73:1507-1525.

4. Riley $\mathrm{G}$. The pathogenesis of tendinopathy. A molecular perspective. Rheumatology (Oxford) 2004; 43:131-142.

5. Wang JH, losifidis MI, Fu FH. Biomechanical basis for tendinopathy. Clin Orthop Relat Res 2006;443:320-332.

6. Bi Y, Ehirchiou D, Kilts TM, Inkson CA, Embree MC, et al. Identification of tendon stem/progenitor cells and the role of the extracellular matrix in their niche. Nat Med. 2007; 13:1219-1227.

7. Zhang J, Wang JH. Characterization of differential properties of rabbit tendon stem cells and tenocytes. BMC Musculoskelet Disord. 2010;11:10-20.

8. Rui YF, Lui PP, Li G, Fu SC, Lee YW, et al. Isolation and characterization of multipotent rat tendon-derived stem cells. Tissue Eng Part A. 2010;16:1549-1558.

9. Zhou Z, Akinbiyi T, Xu L, Ramcharan M, Leong DJ, et al. Tendon-derived stem/progenitor cell aging: defective self-renewal and altered fate. Aging Cell. 2010; 9:911-915. 
10. Zhang J, Wang JH. Mechanobiological response of tendon stem cells: implications of tendon homeostasis and pathogenesis of tendinopathy. J Orthop Res. 2010; 28:639-643.

11. Chang J, Thunder R, Most D, Longaker MT, Lineaweaver WC. Studies in flexor tendon wound healing: neutralizing antibody to TGF-beta1 increases postoperative range of motion. Plast Reconstr Surg. 2000;105:148-155.

12. Hsu C, Chang J. Clinical implications of growth factors in flexor tendon wound healing. J Hand Surg Am. 2004;29:551-563.

13. Molloy $T$, Wang $Y$, Murrell $G$. The roles of growth factors in tendon and ligament healing. Sports Med. 2003;33:381-394.

14. Wang JH. Mechanobiology of tendon. J Biomech. 2006;39: 1563-1582.

15. Foster TE, Puskas BL, Mandelbaum BR, Gerhardt MB, Rodeo SA. Platelet-rich plasma: from basic science to clinical applications. Am J Sports Med. 2009;37:2259-2272.

16. Murray MM, Spindler KP, Abreu E, Muller JA, Nedder A, et al. Collagen-platelet rich plasma hydrogel enhances primary repair of the porcine anterior cruciate ligament. J Orthop Res. 2007;25:81-91.

17. Mishra A, Pavelko T. Treatment of chronic elbow tendinosis with buffered platelet-rich plasma. Am J Sports Med. 2006;34: 1774-1778.

18. Sanchez M, Anitua E, Azofra J, Andia I, Padilla S, et al. Comparison of surgically repaired Achilles tendon tears using plateletrich fibrin matrices. Am J Sports Med. 2007; 35:245-251.

19. de Vos RJ, Weir A, van Schie HT, Bierma-Zeinstra SM, Verhaar JA, et al. Platelet-rich plasma injection for chronic Achilles tendinopathy: a randomized controlled trial. JAMA. 2010;303: 144-149.

20. Zhang J, Wang JH. The effects of mechanical loading on tendons - an in vivo and in vitro model study. PLoS One. 2013;8: e71740.

21. Vogel G. Stem cells. Diseases in a dish take off. Science 2010;330:1172-1173.

22. Tiscornia G, Vivas EL, Belmonte JC. Diseases in a dish: modeling human genetic disorders using induced pluripotent cells. Nat Med. 2011; 17:1570-1576.
23. Padulo J, Oliva F, Frizziero A, Maffulli N. Muscle, Ligaments and Tendons Journal. Basic principles and recommendations in clinical and field science research. MLTJ. 2013;4:250-252.

24. Zhang J, Wang JH. Platelet-rich plasma releasate promotes differentiation of tendon stem cells into active tenocytes. Am J Sports Med. 2010;38:2477-2486.

25. Anitua E, Andia I, Sanchez M, Azofra J, del Mar Zalduendo $\mathrm{M}$, et al. Autologous preparations rich in growth factors promote proliferation and induce VEGF and HGF production by human tendon cells in culture. J Orthop Res. 2005;23:281286.

26. Emans PJ, Spaapen F, Surtel DA, Reilly KM, Cremers A, et al A novel in vivo model to study endochondral bone formation; HIF-1alpha activation and BMP expression. Bone 2007; 40:409-418.

27. Boykiw R, Sciore P, Reno C, Marchuk L, Frank CB, et al. Altered levels of extracellular matrix molecule mRNA in healing rabbit ligaments. Matrix Biol. 1998;17:371-378.

28. Engebretsen L, Steffen K, Alsousou J, Anitua E, Bachl N, et al. IOC consensus paper on the use of platelet-rich plasma in sports medicine. Br J Sports Med. 2010;44:1072-1081.

29. Zhang J, Wang JH. The effects of mechanical loading on tendons - an in vivo and in vitro model study. PLoS One. 2013;8: e71740.

30. Zhang J, Middleton KK, Fu FH, Im HJ, Wang JH. HGF mediates the anti-inflammatory effects of PRP on injured tendons. PLoS One. 2013;8:e67303.

31. Gill TJ, Carroll KM, Hariri S. Open Patellar Tendon Debridement for Treatment of Recalcitrant Patellar Tendinopathy: Indications, Technique, and Clinical Outcomes After a 2-Year Minimum Follow-up. Sports Health: 2013;5:276-280.

32. Witt BL, Hyer CF. Achilles tendon reattachment after surgical treatment of insertional tendinosis using the suture bridge technique: a case series. J Foot Ankle Surgery. 2012;51:487493.

33. Alfredson $\mathrm{H}$, Cook J. A treatment algorithm for managing Achilles tendinopathy: new treatment options. $\mathrm{Br} \mathrm{J}$ Sports Med. 2007;41:211-216. 